\title{
Randomization of Clinical Trial Participants via an Integrated Web Service
}

\author{
Panos BONOTIS ${ }^{\mathrm{a}}$, Achilleas CHYTAS ${ }^{\mathrm{a}}$, Giorgos ZACHARIOUDAKIS ${ }^{\mathrm{b}}$, Christina \\ KARAMANIDOU ${ }^{\mathrm{a}}$, Lefteris KOUMAKIS ${ }^{\mathrm{b}}$, Kostas STAMATOPOULOS $^{\mathrm{a}}$ and \\ Pantelis NATSIAVAS ${ }^{\mathrm{a}, 1}$ \\ anstitute of Applied Biosciences, Centre for Research \& Development Hellas, \\ Thessaloniki, Greece \\ ${ }^{\mathrm{b}}$ Institute of Computer Science, Foundation for Research \& Technology Hellas, \\ Heraklion, Greece
}

\begin{abstract}
Randomization is an inherent part of Randomized Clinical Trials (RCTs), typically requiring the split of participants in intervention and control groups. We present a web service supporting randomized patient distribution, developed in the context of the MyPal project RCT. The randomization process is based on a block permutation approach to mitigate the risk of various kind of biases. The presented service can be used via its web user interface to produce randomized lists of patients distributed in the various study groups, with a variant block size. Alternatively, the presented service can be integrated as part of wider IT systems supporting clinical trials via a REST interface following a micro-service architectural pattern.
\end{abstract}

Keywords. block permutation, randomized clinical trial, electronic data capture

\section{Introduction}

Random allocation of participants to different groups is an inherent part of Randomized Clinical Trials (RCTs). Typically, these groups are the so-called "intervention group" (the group of patients which are actually monitored to get insights for the respective intervention - e.g., the test of a drug under development) and the so-called "control group" (the group of patients under standard treatment monitored in order to provide a comparison baseline for the intervention). Random allocation of RCT participants to the respective groups is considered as "best practice" to mitigate potential biases [1]. Block permutation is the most prominent algorithmic approach defining blocks of specific size and then randomizing the elements of each specific block [2]. While, there are online tools available these typically lack the ability to automatically integrate in wider IT systems that might support RCTs (e.g. Electronic Data Capture - EDC systems) or they are based on legacy communication interfaces which are not actively maintained [3].

Electronic Patient Reported Outcomes (ePROs) paradigm is actively investigated in terms of palliative care [4]. To this end, MyPal project2 includes two clinical studies

\footnotetext{
${ }^{1}$ Corresponding Author: Pantelis Natsiavas, eHealth Lab, INAB-CERTH, 6th Km. Charilaou-Thermi Road, Thermi, Thessaloniki, Greece; E-mail: pnatsiavas@certh.gr.

${ }^{2} \mathrm{MyPal}$ has received funding from the European Union's Horizon 2020 research and innovation programme under grant agreement No. 825872 . For more information on the project visit: https://mypalproject.eu/\}
} 
aiming to evaluate the application of ePRO tools [5]. The presented service is part of MyPal IT infrastructure but it also can be used independently.

\section{Methods \& Results}

The service supports the assignment of a single participant or a list of participants (size $\mathrm{N})$ to either the "intervention" or "control" group, based on the allocation percentage (input variable AP) and the block size (input variable BS). For N participants, an N/BS number of blocks are created and, in each block, participants are randomly distributed satisfying the AP requirement. Beyond block permutation, the presented service also supports various randomization schemes based on various statistical distributions. The provided functionalities are available via both the web UI and the REST API, supporting also RCTs with multiple clinical sites with centralized randomization schemes (Figure $1)$.

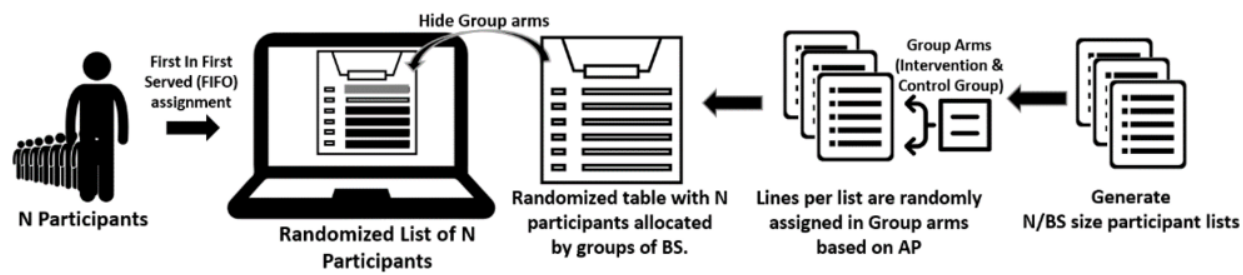

Figure 1. Randomization logic.

\section{Discussion}

Randomized allocation of participants in the various groups of RCTs has been identified as a key process to mitigate potential biases in terms of patient recruitment and allocation. In terms of regulation, the context is vague as there are not widely accepted technical requirements. In this poster, we present a service enabling randomized allocation of patients to RCT groups, able to be used independently via its web UI or via a REST communicating interface. We argue that providing two ways of communication might be crucial as RCTs could use ICT systems to manage patients or alternatively manage patients using a less systematic manner (via excel files, or even manually).

\section{References}

[1] Lim CY, In J. Randomization in clinical studies. Korean J. Anesthesiol. June 2019;72(3):221-232.

[2] Efird J. Blocked randomization with randomly selected block sizes. Int. J. Environ. Res. Public Health. Jan. 2011;8(1):15-20.

[3] Schrimpf D et al. Possible combinations of electronic data capture and randomization systems: Principles and the realization with RANDI2 and OpenClinica. Methods Inf. Med. 2014;53(3):202-207.

[4] Karamanidou $\mathrm{C}$ et al. Electronic patient-reported outcome-based interventions for palliative cancer care: A systematic and mapping review. JCO Clin. Cancer Informatics. 2020;4.

[5] Maramis C et al. Using Electronic Patient Reported Outcomes to Foster Palliative Cancer Care: The MyPal Approach. in Proceedings - 2019 IEEE 19th International Conference on Bioinformatics and Bioengineering, BIBE 2019. 2019;405-409. 